80 Tropelías. Revista de Teoría de la Literatura y Literatura Comparada, número extraordinario 5 (2019) Iris Llop Mangas

\title{
KUNDERA LECTOR DE BROCH: LA NOVELA COMO FORMA DE CONOCIMIENTO
}

\author{
KUNDERA AS BROCH'S READER: \\ THE NOVEL AS A FORM OF KNOWLEDGE
}

Iris LLOP MANGAS

Universitat de Barcelona

iris.1lop@ub.edu

\begin{abstract}
Resumen: El propósito del presente estudio consiste en analizar la noción de «ensayo específicamente novelesco» desarrollada por Milan Kundera en sus ensayos. Dicha noción aparece por primera vez en L'art du roman (1986) ligada a la lectura que Kundera propone de Die Schlafwandler de Hermann Broch y se nutre de la concepción de la novela como instrumento de conocimiento del escritor austríaco. Los desplazamientos semánticos y las modificaciones operadas por Kundera para adaptar la poética de Broch a su idea de novela dan lugar a una concepción de la función del ensayo en la novela que permite entender este género como una forma de reflexión alternativa al modelo científico o filosófico de pensamiento
\end{abstract}

Palabras clave: Kundera, Broch, Novela, Ensayo, Conocimiento.

Abstract: The purpose of the present study is to analyse the notion of «specifically novelistic essay» developed by Milan Kundera in his essays. This notion appears for the first time in The Art of the Novel (1986) linked to his interpretation of The Sleepwalkers from Hermann Broch and follows Broch's conception of the novel as a form of knowledge. The semantic shifts and alterations of Broch's novel poetics allow Kundera to adapt this model to his own idea of the novel, characterized by the new function of the essay form in the novel, which allows us to understand the novel as a form of reflection that can be an alternative to the scientific or philosophical way of thinking.

Keywords: Kundera, Broch, Novel, Essay, Knowledge. 

testaments trahis y Le rideau) se construye a partir de las lecturas que ofrece de sus modelos literarios. De este modo, Kundera presenta su idea de novela a la vez que se inserta en una tradición estética que privilegia las «novelas que piensan». En el capítulo de L'art du roman (1986) dedicado a Die Schlafwandler (1933) de Hermann Broch, Kundera describe por primera vez el arte del «ensayo específicamente novelesco» como un tipo de ensayo que aporta un mensaje de forma hipotética, lúdica o irónica.

Si bien la noción de «ensayo novelesco» se construye a partir de la lectura de un corpus diverso de novelas, especialmente las pertenecientes a la tradición centroeuropea representada por Hermann Broch, Franz Kafka y Robert Musil, y se fundamenta en el pensamiento experimental y aforístico de Nietzsche, la teoría de la novela que Broch presenta en sus cartas y ensayos es el punto de partida recurrente de la concepción kunderiana de la novela como forma de reflexión. La pregunta que guía la presente investigación es cuál es el papel que juega el pensamiento de Broch en el desarrollo de la noción de «ensayo específicamente novelesco» y qué referencias implícitas nos descubre la filiación de Kundera con la teoría brochiana.

Para llevar a cabo este estudio, en primer lugar, presentaremos brevemente las ideas que Broch expone en sus cartas, conferencias y ensayos acerca de la capacidad cognoscitiva de la novela y la forma que permite desarrollar este tipo de conocimiento. Así, se expondrá qué entiende Broch por novela gnoseológica o cognoscitiva («erkenntnistheoretischer Roman») y por novela polihistórica («polyhistorischer Roman»). A continuación, comentaremos la lectura que Kundera propone de estos textos y de la trilogía Die Schlafwandler para, finalmente, ver las trazas de esas lecturas en su propuesta de un pensamiento específicamente novelesco.

Debido a que situamos el foco de la investigación en la noción acuñada por Kundera, no pretendemos aquí ofrecer una descripción exhaustiva de la teoría de Broch, sobre la cual se puede consultar una extensa bibliografía específica. Lo que se propone es un recorrido por el nacimiento y desarrollo de algunas categorías clave para comprender la relación entre novela y conocimiento desde la perspectiva de Kundera.

\section{Los ideales de la novela brochianos: «erkenntnistheoretischer Roman» $\mathbf{y}$ «polyhistorischer Roman»}

Podemos distinguir dos grandes líneas de reflexión en los textos de Broch: la que se ocupa de la estética de la novela y se pregunta por su función y sus posibilidades como forma de conocimiento y, en segundo lugar, pero en estrecha relación con la primera, la que se compone de las propuestas sobre la forma de la novela, esto es, sobre los recursos lingüísticos que permiten concretar el ideal estético de este género. 
Autores de estudios previos sobre la estética de Broch como Christine Mondon (2011, pp. 4782), quien revisa las aportaciones de los principales intérpretes de Broch como Manfred Durzak (1978), Paul Michael Lützeler (1987), Hartmut Steinecke (1968) y Monika Ritzer (1988), han usado un criterio cronológico para entender los cambios sucesivos que el autor austríaco introduce en las definiciones de las ideas centrales que rigen su concepción de la novela. De acuerdo con esta tradición, nos limitaremos aquí a estudiar los textos que se gestan durante la redacción de la trilogía de Die Schlafwandler, obra que guía la lectura de Kundera. Las cartas escritas entre 1928 y 1933, las conferencias «James Joyce und die Gegenwart» (James Joyce y el presente) y «Das Weltbild des Romans» (La cosmovisión de la novela) de $1932^{1}$ serán la principal fuente del pensamiento estético de Broch.

De acuerdo con estos estudios, la noción de «erkenntnistheoretische Roman», traducida como novela epistemológica, pero también como gnoseológica, es una idea general en la estética brochiana que no designa prácticas literarias concretas, sino la razón de ser o la función específica de la novela como género. La tarea epistemológica de la novela la alejaría de tendencias corruptas del arte como el kitsch o el arte por el arte que, según Broch, olvidan el componente ético de todo acto artístico.

En las cartas que Broch intercambia con su editor de la Rhein-Verlag, Dr. Daniel Brody, durante la escritura de su trilogía, se recogen algunas de las primeras formulaciones de la novela epistemológica. En una carta del 16 de julio de 1930 dirigida a Daisy Brody, esposa del editor, expone lo siguiente:

[...] lo que sólo se insinúa en Los Sonámbulos es algo que no va en la dirección de Joyce [...], es decir, la novela epistemológica en lugar de la psicológica, es decir, la novela en la que la motivación psicológica se basa en actitudes básicas epistemológicas y en la lógica y plausibilidad real de los valores. (Broch, 1957, p. 23) ${ }^{2}$

La necesidad de poner nombre a los distintos tipos de novela surge de la voluntad de distinguir diferentes modelos narrativos que Broch observa cuando compara su obra con la de James Joyce, uno de sus principales referentes. Si bien solo disponemos de una parte de la correspondencia, sabemos que Broch construye su propia idea de novela a partir de un doble movimiento de imitación y distanciamiento de la obra de Joyce, como vemos en el fragmento que comenta con la señora Brody; Broch muestra su desinterés por el análisis psicológico de los personajes que lee en Joyce pero se interesa por cómo el irlandés relaciona valores abstractos con el comportamiento de los personajes.

De esta forma, Broch nos ofrece una primera clave de lectura -que seguirá el propio Kunderaacerca de la función de los personajes en la tarea epistemológica que lleva a cabo la novela. Cada uno

\footnotetext{
${ }^{1}$ La conferencia «James Joyce und die Gegenwart» se pronuncia en 1932 pero la versión publicada en las obras completas es de 1936.

${ }^{2}$ Dado que no existe versión castellana de la correspondencia de Broch y a no ser que se indique lo contrario, las traducciones que se ofrecen son mías. Se facilitará en estos casos la versión original en la nota al pie. «was in den Schlafwandlern erst angedeutet ist, ist ja doch etwas, das nicht in der Richtung Joyce liegt [...], nämlich der erkenntnistheoretische Roman statt des psychologischen, d. h. der Roman, in dem hinter die psychologische Motivation auf erkenntnistheoretische Grundhaltungen und auf die eigentliche Wertlogik und Wertplausabilität zurückgegangen wird.»
} 


\section{Kundera lector de Broch: la novela como forma de conocimiento}

de los protagonistas (Pasenow, Esch y Huguenau) sirve para descubrir distintas actitudes frente a la degradación de los valores que se representa en Die Schlafwandler. En una fechada carta pocos días más tarde (26 de julio de 1930), Broch sigue ahondando en estas ideas, a partir de otro fragmento de Joyce.

[...] detrás de lo psicológico está probablemente lo epistemológico, y más allá de eso no se puede ir racional y cognitivamente. En lo irracional y productivo, sin embargo, hay más capas detrás de la plausibilidad epistemológica, capa tras capa llenas de mitos, y Joyce ya ha llegado allí. (Broch, 1957, p. 27) ${ }^{3}$

Aquí se esboza una de las grandes preocupaciones estéticas de Broch, si no la más importante: cómo representar lo irracional en el arte y, más concretamente, cómo mostrar las motivaciones irracionales detrás de las acciones humanas. La categoría de «erkenntnistheoretische Roman» tiene que ver pues con un tipo de novelas que ofrecen un conocimiento sobre la acción humana que va más allá de las explicaciones científicas o psicológicas, basadas en la racionalidad. Este conocimiento, siguiendo el modelo de Joyce, estaría relacionado con la construcción los mitos, a menudo usados como forma de representación de determinadas visiones del mundo en conflicto entre ellas para lograr una hegemonía.

En la conferencia «Das Weltbild des Romans», Broch desarrolla esta relación entre novela y mito y se pregunta por aquellos elementos que dan forma a una cosmovisión y qué tipo de cosmovisión puede representar la novela. A la primera pregunta responde que la cosmovisión se basa en unos valores concretos y, por lo tanto, la visión del mundo específica de la novela estará determinada por sus valores. También añade que toda cosmovisión parcial del mundo tiene la ambición de cambiar el estado actual del mismo, algo que constituye su exigencia ética. Pero ¿en qué consiste esta exigencia ética del arte? Según Broch, la creación artística es «siempre un acto de modulación, de dar forma; es, por consiguiente, un resultado estético, en el más amplio sentido de la palabra. ¡Acto ético y resultado estético!» (Broch, 1974, p. 267) ${ }^{4}$. El antimodelo de esta forma novelesca, como hemos comentado, sería el arte kitsch debido a que éste «lo sacrifica todo en aras del efecto estético» y confunde la «categoría ética con la estética» (Broch, 1974, pp. 272-274).

A partir del análisis de las cosmovisiones de la novela de autores admirados por Broch como Balzac, Zola y Dostoievski, el escritor vienés observa algunas limitaciones en la visión del mundo que este género puede construir. Al final de la conferencia, Broch afirma que «la novela no es capaz de presentar el mundo en su auténtica realidad porque nunca podrá asumir la tarea de la ciencia. Hemos visto que la novela no tiene derecho a presentar el mundo tal como debería ser porque quedaría en novela tendenciosa» (Broch, 1974, p. 294). Ante estas limitaciones, cabe preguntarse qué le queda a la novela, cuál es su razón de ser:

\footnotetext{
${ }^{3}$ «[...] hinter dem Psychologischen steht wohl das Erkenntnistheoretische, und darüber lässt sich rational und erkennend nicht hinausgehen. Im Irrationalen und Produktiven aber stehen hinter der erkenntnistheoretischen Plausibilität noch weitere Schichten, Schicht um Schicht voller Mythos, und dort ist Joyce schon angelangt.»

${ }^{4}$ «[...] das Resultat ist immer ein Akt der Formung und Formgebung, ist also, im weitesten Sinne gesprochen, ein ästhetisches Resultat. Etischer Akt und ästhetisches Resultat.» (Broch, 1975, p. 90)
} 
Sí, la novela ha de ser espejo de todas las demás visiones del mundo, pero estas visiones son para ella trozos de la realidad, exactamente igual que cualquier otra visión parcial de la realidad visible. Y de la misma manera que todas y cada una de las parcelas de la realidad que la novela recoge del mundo exterior, también ella tiene su propia estructura. (Broch, 1974, p. 295)

Así, la tarea de la novela es entretejer en la narración las distintas visiones del mundo, marcadas por sus correspondientes valores fundacionales y a menudo representadas por los distintos personajes que protagonizan la acción, como ya había sugerido a partir de sus análisis de los caracteres en Joyce.

La concreción formal de esta concepción de la novela vendrá de la mano de la siguiente noción: la «novela polihistórica». Esta categoría, a diferencia de la anterior, designa los procedimientos textuales concretos a partir de los cuales se puede desarrollar un conocimiento sobre lo irracional de la conducta humana y sobre las distintas cosmovisiones que se derivan de los valores que orientan las acciones de los personajes. Uno de los primeros usos de esta denominación lo encontramos en una carta de Broch a su editor el 5 de agosto de 1931:

Usted conoce mi teoría: mi teoría: la novela y la nueva forma novelesca están encargadas de ingerir esas partes de la filosofía que corresponden, ciertamente, a necesidades metafísicas, pero que teniendo en cuenta el estado actual de la investigación deben ser consideradas hoy en día como "no científicas" o, como dice Wittgenstein, como "místicas". La época de la novela polihistórica ha comenzado. Pero no se trata de forzar este polihistoricismo en el libro bajo la forma de discursos "cultos" o, para poder alojarlo, preferir hombres de ciencia como héroes de la novela. La novela es poesía, tiene que ver con los impulsos motrices primitivos del alma [Ur-Moventien] y elevar una capa social "cultivada" a la categoría de soporte de la acción novelesca es transformarla en Kitsch. (Broch, 1957, p. 59) ${ }^{5}$

Según Harmut Steinecke (1968, p. 28) el adjetivo polihistórico apunta a la herencia filosófica de la novela, pues comparte con ella el anhelo de capturar la totalidad del saber sobre el mundo. Dando prioridad a la segunda parte de la cita, Karl Robert Mandelkow (1962, p. 156) interpreta la noción de «polihistoricismo» como aquella que designa una tipología específica novelas que tienen por objetivo presentar un conocimiento gracias a la unión de ciencia y literatura, y de reflexión y representación en la obra de arte. El gran reto en estas obras es pues, según Mandelkow, mantener el equilibrio entre el pensamiento abstracto de las reflexiones, que permite el conocimiento sobre el mundo, y la concreción de la representación literaria de ese mundo.

Ligados a la noción de «polihistoricismo» aparecen en esta carta diferentes elementos a tener en cuenta en relación a la idea de novela de Broch: en primer lugar, expone de nuevo su tarea, ocuparse de lo místico e irracional, en oposición a aquellas propias de la filosofía o la ciencia. La novela explora pues los «Ur-Moventien», los motivos previos a la razón que impulsan los actos de los personajes. Para mostrar como se lleva a cabo, Broch expone primero un ejemplo negativo: no se trata pues de representar en la novela diálogos cultos entre personajes ilustrados -en la misma carta cita como

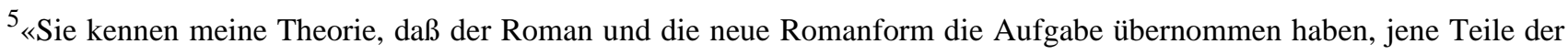
Philosophie zu schlucken, die zwar metaphysischen Bedürfnissen entsprechen, gemäß dem derzeitigen Stande der Forschung aber heute als «unwissenschaftlich» oder, wie Wittgenstein sagt, als «mystisch» zu gelten haben. Die Zeit des polyhistorischen Romans ist angebrochen. Es geht aber nicht an, daß man diesen Polyhistorismus in Gestalt «gebildeter» Reden im Buche unterbringt oder zu dieser Unterbringung Wissenschaftler als Romanhelden präferiert. Der Roman ist Dichtung, hat also mit den Ur-Moventien der Seele zu tun, und eine «gebildete» Gesellschaftsschicht zum Romanträger zu erheben, ist eine absolute Verkitschung.»
} 
ejemplos de este caso las reflexiones sobre la escritura en Les faux-monnayeurs de Gide o las conversaciones entre Castorp, Settembrini y Naphta en Der Zauberberg de Mann. Lo que persigue la novela polihistórica es representar la diversidad de visiones del mundo y el origen las mismas a través de la acción de los personajes y sus interrelaciones.

Broch, siguiendo este modelo en el que «lo científico no se utiliza como fuente de conversación, sino que nada y resuena como la capa racional más alta» (Broch, 1957, p. 59), concibe la arquitectura de la trilogía Die Schlafwandler de forma que las distintas líneas argumentales lleven a representar las distintas visiones del mundo que representa cada personaje. Esto es especialmente relevante en el tercer volumen, Huguenau oder die Sachlichkeit, donde se encuentran los personajes principales de los libros anteriores, Pasenow y Esch, con el protagonista del tercero, Huguenau. Paralelamente a las acciones de estos personajes encontramos dos relatos más, aparentemente no relacionados con la acción de los protagonistas: «La historia de la chica salutista de Berlín» (aquí referida como la del «Ejército de Salvación») y la «Degradación de los valores». ¿Cómo se consigue pues la unidad compositiva entre estos fragmentos? Según Broch (1957, p. 59) es necesaria una composición en contrapunto continuo, de forma que cada capítulo establezca una relación de comentario con el que le precede y el siguiente, aunque no forme parte de la misma línea argumental. Para lograrlo, una de las estrategias se centra en la relación entre los personajes y sus relatos: así Bertrand Müller, el narrador del «Ejército de Salvación» se revela como autor del texto sobre la degradación de los valores, aunque es algo que no descubrimos hasta la página 146 de la novela.

La composición contrapuntística necesita pues, sobre el vínculo argumental entre los personajes, un segundo vínculo basado en los temas principales tratados por las distintas historias. Así, aunque personajes como Bertrand y Esch no coincidan en ninguna escena, ambos se interrogan sobre cómo actuar delante de la degradación y pérdida de sentido de los valores con los que se identificaban y en los que basaban sus acciones.

\section{Kundera lector de Broch: interpretaciones de una poética de la novela}

La relación entre el pensamiento de Broch y Kundera sobre la novela ha sido objeto de investigaciones previas a partir de la publicación de L'art du roman (1986) y Les testaments trahis (1993), si bien Jean-Michel Rabaté (1983) ya había apuntado antes el papel de Kundera como introductor de la obra de Broch en el campo literario francés, una aproximación en la que ahonda Isabelle Gabolde en La lecture comme pratique cognitive et devoir d'éveil (2010). Los estudios de Liisa Steinby (née Saariluoma, 1999) y Paola Gambarota (1999) son los primeros en exponer el uso que el novelista checo hace en sus ensayos del pensamiento literario de Broch. Steinby ampliará su estudio con las reflexiones aportadas por Kundera en Le rideau (2005) en su libro Kundera and Modernity (2013).

Según Steinby y Gambarota, existen dos vínculos entre el pensamiento de Kundera y el de Broch: la función epistemológica de la novela y la preocupación por como la composición de la novela 
contribuye al desarrollo de esta esta función, gracias a la unión temática y de las relaciones entre las cosmovisiones entre los personajes. Para poder entender los desplazamientos semánticos y conceptuales que Kundera hace con las ideas de Broch, analizaremos la evolución del papel de éste en la construcción del pensamiento de Kundera en sus sucesivos ensayos.

Del mismo modo que los conceptos acuñados por Broch nacen inspirados por sus lecturas de la obra de Joyce o Goethe, Kundera desarrolla sus ideas sobre la novela a partir de su exegesis de Die Schlafwandler. En el primer capítulo de L 'art du roman, Kundera atribuye a Broch una frase que citará en múltiples ocasiones: «descubrir lo que sólo una novela puede descubrir es la única razón de ser de una novela» (Kundera, 2007, p. 16). Dicha sentencia, si bien no describe el objeto de conocimiento de la novela sí que nos ilustra sobre la naturaleza de su actividad: el descubrimiento. La novela nos presenta de otro modo la realidad que percibimos cotidianamente, siguiendo las palabras de Broch, recogiendo las parcelas de realidad y construyendo con ellas la narración.

Kundera se presenta de este modo como heredero de la preocupación de Broch por la especificidad de la novela y será a partir de la lectura de Die Schlafwandler bajo la luz de las ideas de su autor como Kundera introduce su perspectiva sobre cómo la novela puede comunicar ese conocimiento específico sobre el mundo. En el capítulo «Notas inspiradas por Los sonámbulos», analiza en primer lugar la composición de la novela y la construcción de los personajes, es decir, los aspectos formales de arquitectura de la obra brochiana, para luego preguntarse por la relación de esas opciones estéticas con los textos teóricos del autor.

Kundera familiariza a su lector con las distintas líneas argumentales entrelazadas que teje Broch para mostrar esas distintas cosmovisiones, para después centrarse en el análisis de los tres personajes principales: Pasenow, Esch y Huguenau, concebidos no como encarnaciones de caracteres reales sino como tres posibilidades, tres tomas de posición frente a la degradación de los valores. Así, Pasenow percibe la falta de sentido de los valores que encarna, pero a pesar de ello los lleva hasta las últimas consecuencias; Esch ve los valores vacíos de contenido, solo es capaz de distinguir el imperativo tras el valor; Huguenau, finalmente, ya no es consciente de la perdida de los valores comunes y vive encontrando siempre una justificación convincente y satisfactoria para sus actos, aunque sean criminales. Kundera añade al lado de las posibilidades de Broch, las de K en Das Schloss de Kafka y el Soldado Schwejk de Hašek, como personajes que también afrontan la pérdida de un sistema de valores comunes, compartidos por la sociedad, de tal modo que todos ellos forman «cinco puntos de orientación sin los cuales me parece imposible perfilar el mapa existencial de nuestro tiempo» (Kundera, 2007, p. 73). Dar cuenta de estos mapas de posibilidades existenciales es, según Kundera, la razón de ser de la novela.

Dicha exploración existencial se concreta, según el novelista checo, en la construcción de personajes cuyas acciones no responden a una lógica causal y que proponen una de las preguntas fundamentales de la epistemología de la novela: «¿Qué es el acto? [...] ¿Cómo nace una decisión? ¿Cómo se transforma en acto y cómo los actos se encadenan para convertirse en aventura?» (Kundera, 2007, p. 77). La pregunta por el ser del hombre se inicia en esa exploración de la acción y su origen. 
Para Kundera, uno de los descubrimientos de Broch es «el papel que lo irracional desempeña en nuestras decisiones, en nuestra vida. [...] La lógica irracional se funda en el mecanismo de la confusión» (Kundera, 2007, p. 79) Pero ¿cómo se llega a mostrar ese aspecto irracional en Die Schlafwandler? Kundera expone un primer ejemplo a partir del primer libro, Pasenow und die Romantik:

Pasenow tiene un escaso sentido de la realidad; se le escapa la causa de los acontecimientos; nunca sabrá lo que se oculta tras a mirada de los demás; sin embargo, aunque encubierto, irreconocible, a-causal, el mundo exterior no permanece mudo: le habla. Es como en el célebre poema de Baudelaire en el que «los largos ecos...confunden», en el que «los perfumes, los colores y los sonidos se responden»: una cosa se aproxima a otra, se confunde con ella (Elisabeth se confunde con la Virgen) y de este modo, mediante este acercamiento, se explica. (Kundera, 2007, p. 80)

En su trilogía, Broch nos presenta a unos personajes que actúan supuestamente de acuerdo con un sistema de valores ligado a su profesión («el contable Esch» o el «albañil Gödicke»), como último recurso de valores compartidos, como si esta posición social nos pudiese orientar a la hora de anticipar sus acciones. Pero este andamiaje de valores empieza a tambalear ante la incomprensión entre los personajes, una incomprensión que también experimentan los personajes respecto a sus propias acciones. Huguenau, Esch o el mayor Pasenow olvidan fácilmente el propósito de sus acciones o la impresión que se habían formado de otros personajes: de esta forma cobra sentido el título de la trilogía, pues los personajes se relacionan entre ellos y con el mundo como sonámbulos, con una conciencia dudosa de lo que les rodea y de sí mismos. Como dice Kundera, hay que

[...] detenerse en las acciones tanto ilógicas como comprensibles, para ver un orden oculto, subterráneo, sobre el que se fundan las decisiones [...] Estos personajes no son capaces de afrontar la realidad como algo concreto. Ante sus ojos todo se transforma en símbolos [...] y es a los símbolos a los que reaccionan cuando creen actuar sobre la realidad. [...] Broch nos hace comprender que el sistema de las confusiones, el sistema del pensamiento simbólico, está en la base de todo comportamiento tanto individual como colectivo. (Kundera, 2007, p. 81)

La lectura de Kundera se centra pues, en primer lugar, en la construcción de los personajes y, en segundo lugar, en la arquitectura de la novela que deriva o que aparece como necesaria para representar esas distintas posibilidades existenciales ligadas a cada personaje.

Como hemos visto hasta ahora, la idea de la novela como forma de conocimiento queda apuntada al inicio de $L$ 'art du roman y se va consolidando a partir de las lecturas de Broch que propone Kundera. Por ello sorprende la poca atención que dedica a las connotaciones que el austríaco da a las nociones de novela epistemológica y polihistórica, hasta el punto de presentarlas como sinónimas («Broch rechaza la estética de la novela «psicológica» y le opone la novela que él denomina «gnoseológica» $\mathrm{o}$ «polihistórica» Kundera, 2007, p. 84) aunque manifiesta su preferencia por la primera debido a que bajo su criterio la segunda puede llevar a equívocos. El problema de comprensión que aduce se basa en las connotaciones negativas que el mismo Kundera (2007, p. 84) atribuye al relato de la Historia en su relación con el de la novela: para él una novela polihistórica sería la que da cuenta del saber humano en un momento histórico determinado, una suerte de novela enciclopédica, que se aleja de la razón de ser de la novela: el conocimiento sobre el hombre y su comportamiento, no saber del hombre sobre 
esa realidad histórica dada. Kundera desplaza así el significado que Broch da a la noción de polihistoricismo en «Das Weltbild des Romans» y la adapta a la idea de novela que él está construyendo.

[Broch] sabe igualmente que la novela es capaz de integrar tanto la poesía como la filosofía sin perder por ello nada de su identidad, que se caracteriza (basta con recordar a Rabelais y Cervantes) por su tendencia a abarcar otros géneros, a absorber los conocimientos filosóficos y científicos. En la óptica de Broch, pues, el término «polihistórico» quiere decir: movilizar todos los medios intelectuales y todas las formas poéticas para esclarecer «lo que únicamente la novela puede descubrir»: el ser del hombre. (Kundera, 2007, p. 84)

A pesar de presentarlo como una descripción del pensamiento de Broch, en parte gracias a la insistencia en las citaciones, por otro lado, no referenciadas, nos encontramos delante de una apropiación y desplazamiento del significado de las palabras, algo que Kundera llevará a cabo en otros momentos de su ensayo para presentarse como continuador del legado de Broch. Así, parte de reflexiones atribuibles al autor austríaco, como el hecho que la novela es capaz de integrar otros discursos, para después ejercer cierta violencia sobre el sentido que le otorga Broch, quien no habla de absorber los conocimientos de la filosofía o la ciencia, sino que la literatura debía asumir una tarea para la que ni la filosofía ni la ciencia tenían recursos para representar: la idea la totalidad o el orden del mundo a través del mito. Kundera, como nos recuerdan Steinby (2013, p. 78) y Gambarota (1999, p. 54), considera la novela un género caracterizado por el juego y el escepticismo, que pone de manifiesto la relatividad de las verdades.

La distancia entre ambos autores se manifiesta también en la lectura que Kundera ofrece de Die Schlafwandler, pues es justamente a partir de sus insatisfacciones, lo que él llama "lo incumplido" que el autor checo construye su idea de novela y entra en el debate estético con las propuestas del austríaco. Según Kundera, hay tres aspectos que Broch no lleva a cabo de forma completa: la intención polifónica de la obra, es decir la claridad arquitectónica y compositiva de la obra, el equilibrio entre las distintas voces y formas lingüísticas y, finalmente, la presentación de un ensayo específicamente novelesco.

Sí bien estas insatisfacciones serán matizadas más tarde en otros ensayos como Les testaments trahis o Le rideau, detengámonos en las posibilidades que abre esta crítica: observar «lo incumplido» en la obra de Broch permite a Kundera de nuevo presentarse como continuador de un proyecto abierto y establecer una serie de soluciones formales y estéticas a los problemas planteados por Broch. Así, al final de su capítulo dedicado a Die Schlafwandler, Kundera traza las líneas maestras de su idea de novela a partir de tres elementos: el despojamiento radical de la trama como arte de la elipsis, el arte del contrapunto novelesco como forma de diálogo entre las distintas partes y géneros discursivos que componen la novela y un arte del ensayo específicamente novelesco, en el que se transmita un pensamiento hipotético, lúdico e irónico.

\section{El ensayo específicamente novelesco: la novela pensante de Broch y Musil}

Si bien las nociones de elipsis y contrapunto son relevantes para entender la forma que propone Kundera para la novela, siendo el propósito de esta investigación la noción de «ensayo específicamente 
novelesco» y las posibilidades cognoscitivas de la novela, nos centraremos en este tercer elemento destacado por el autor checo. Cabe decir también que, aunque aparecen en relación a la lectura de Broch, las nociones de elipsis y contrapunto son desarrolladas en $L$ 'art $d u$ roman a partir de referentes de la teoría musical y no mediante el análisis de Die Schlafwandler.

Kundera expone sus ideas sobre el papel que juega el ensayo dentro de la novela en su lectura de la obra de Broch, cuando se refiere a los capítulos titulados «Zerfall der Werte» en el tercer volumen de la trilogía. Kundera sostiene que este ensayo sobre la degradación de los valores «aunque presentado como un texto escrito por un personaje, puede ser fácilmente entendido como el razonamiento del autor, como la verdad de la novela, su resumen, su tesis, y alterar así la indispensable relatividad del espacio novelesco» (Kundera, 2007, p. 86).

A pesar de que el mismo Broch había criticado las novelas de tesis o dogmáticas -que presentan el mundo como debería ser- y había advertido de los peligros de los diálogos entre personajes cultos sobre temas filosóficos - poniendo como ejemplo la obra de Mann o Huxley- su ensayo sobre la degradación de los valores no es contrastado por otros puntos de vista, aunque su autoría se atribuya a uno de los personajes. El epílogo de Hugenau oder die Sachlichkeit en el que Broch culmina su reflexión sobre la degradación de los valores a partir del triunfo del personaje inmoral, refuerza el argumento de Kundera sobre la falta de equilibrio entre las distintas perspectivas representadas en la novela.

¿Cuál es pues el modelo de ese ensayo específicamente novelesco? En una de las primeras definiciones Kundera lo presenta como algo que proviene de fuera de la novela, ya sea la filosofía o la ciencia - como nos advertía ya Broch- y que cambia en diálogo con los materiales y el tono de la novela: «al incorporarse a la novela, la meditación cambia de esencia. [...] En el terreno de la novela, no se afirma: es el terreno del juego y de las hipótesis. La meditación novelesca es, pues, esencialmente, interrogativa, hipotética» (Kundera, 2007, p. 99).

Siguiendo esta idea, Kundera ahonda en el vínculo entre reflexión y forma y afirma que el ensayo específicamente novelesco es aquel que pierde su sentido en el momento que queda aislado del contexto de la novela y dentro del «campo magnético del personaje» (Kundera, 2007, p. 101). Para evitar caer en el dogmatismo o en la novela de tesis, Kundera propone una meditación que tiene como objeto «la existencia vista a través de personajes imaginarios» (Kundera, 2007, p. 104) de tal forma que el conocimiento que transmite la novela es siempre parcial, circunscrito al contexto y las coordenadas que caracterizan cada personaje.

Esta concepción del pensamiento tiene raíces nietzscheanas que el mismo Kundera expone en Les testaments trahis (1993). A pesar de recurrir de nuevo a la citación sin referencia, las ideas de Nietzsche que convoca Kundera se encuentran en la obra tardía del filósofo; aforismo dieciséis («Von den Vorurtheilen der Philosophen») de Jenseits von Gut und Böse $e^{6}$ y de la anotación treinta y cinco de

\footnotetext{
${ }^{6}$ «Aunque el pueblo crea que conocer es un conocer-hasta-el-final, el filósofo tiene que decirse: «cuando yo analizo el proceso expresado en la proposición `yo pienso' obtengo una serie de aseveraciones temerarias cuya fundamentación resulta difícil, y tal vez imposible, - por ejemplo, que yo soy quien piensa, que tiene que existir en absoluto algo que piensa, que
} 
mayo-julio de 1885 de los Nachgelassene Fragmente $^{7}$, que Kundera puede desarrollar las características del pensamiento novelesco.

Según el autor checo, «La voluntad de Nietzsche de preservar la «manera efectiva» mediante la cual le llegaron los pensamientos es inseparable de su otro imperativo, que me seduce tanto como el primero: resistirse a la tentación de transformar sus ideas en sistema.» (Kundera, 2007a, p. 163). Para Kundera pues, la reflexión novelesca es aquella capaz de mostrar a partir de una situación y de unos personajes concretos la forma en que nace y se desarrolla una reflexión.

Pero la inspiración nietzscheana no debería confundirnos sobre el tipo de relación entre novela y conocimiento que propone Kundera. Como Broch, el escritor checo combate la idea de una novela filosófica en la que se representen diálogos elocuentes sobre grandes ideas -una opción que considera una servidumbre de la novela a la filosofía- y por ello insiste en la necesidad de distinguir el pensamiento auténticamente novelesco que se caracteriza por ser

[...] asistemático; indisciplinado; está próximo al de Nietzsche; es experimental; fuerza brechas en todos los sistemas de ideas que nos rodean; examina (en particular por mediación de los personajes) todos los caminos de reflexión procurando llegar hasta el final de cada uno de ellos. (Kundera, 2005a, p. 190)

Una vez definidos el tono y las lógicas que rigen el ensayo específicamente novelesco cabe preguntarse cómo toma forma ese pensamiento específico, a partir de qué elementos formales y lingüísticos se construye. Siguiendo la idea de Broch acerca de que el conocimiento de la novela se crea a partir de la belleza, Kundera $(2007$, p. 147) afirma que «la belleza de la reflexión se pone de manifiesto en las formas poéticas de la reflexión» (2007, p. 174): el aforismo, la letanía y la metáfora.

El aforismo lo concibe como la "forma poética de la definición”, mientras que la letanía se inspira en el principio de la repetición en la composición musical: «Quisiera que la novela, en sus pasajes reflexivos, se convirtiera en canto de vez en cuando.» (Kundera, 2007, p. 160). Pero es al papel de la metáfora al que le dedica más atención y la que aparece ligada a la obra de Broch, aunque recurre también a la de Musil.

[...] la metáfora me parece irremplazable como medio para aprehender, iluminada por una repentina revelación, la inasible esencia de las cosas, de las situaciones, de los personajes. La definición de la actitud existencial de Esch: «Él deseaba la claridad sin equívocos: quería crear un mundo de una simplicidad tan clara que su soledad pudiera atarse a esa claridad como a un poste de hierro». Mi norma: muy pocas metáforas en una novela; pero estas deben ser sus puntos luminosos (eventualmente susceptibles de pasar a ser temas que vuelven una y otra vez. (Kundera, 2007, p. 165)

Si el aforismo y la letanía nos presentaban una forma alternativa de definición y una manera de marcar el tono de la reflexión, propios de la novela, la metáfora aparece bajo la mirada de Kundera como la forma de enlazar ideas y crear imágenes que iluminen los enigmas de la existencia que explora la novela, como en el ejemplo de Broch que se acaba de citar, donde la metáfora caracteriza la mirada

\footnotetext{
pensar es una actividad y el efecto causado por un ser que es pensado como causa, que existe un 'yo' y, finalmente, que está establecido qué es lo que hay que designar con la palabra pensar, —que yo sé qué es pensar.» (Nietzsche, 2012, p. 22) 7 «No se debe ocultar y deteriorar el hecho de cómo nos han llegado nuestros pensamientos. Los libros más profundos e inagotables tendrán siempre algo del carácter aforístico e imprevisto de los Pensées de Pascal.» (Nietzsche, 2010, p. 778)
} 
del personaje. Como ya se ha comentado, lo que más interesa a Kundera de Broch es su construcción de los personajes y como presenta cada uno de los protagonistas en contraste con los demás. Comparar las acciones de cada personaje -o sus intentos de actuar de acuerdo con un sistema parcial y problemático de valores- es lo que permite comprender las particularidades de cada sujeto y, al mismo tiempo, entender a complejidad de la existencia que sólo la novela es capaz de representar. Como comenta a partir de Die Schlafwandler,

Para Broch, esta historia está claramente definida como una perpetua degradación de los valores. Los personajes están encerrados en este proceso como en una jaula y deben encontrar el comportamiento adecuado a esta desaparición progresiva de los valores comunes [...] Comprender al hombre arrojado al torbellino de este proceso, comprender sus actitudes y sus gestos, es lo único que cuenta. Broch ha descubierto un territorio desconocido de la existencia. (Kundera, 2007, p. 61)

La lógica propia a través de la cual se construye el ensayo novelesco, vinculado siempre a las vivencias concretas de los personajes, a sus problemas existenciales, es lo que permite no caer en el dogmatismo, en un ensayo que convierta a la novela en tesis, algo que neutralizaría la capacidad de cada personaje para exponer su verdad. Como nos recuerda en Le rideau, el ensayo específicamente novelesco no es un elemento externo añadido a la trama, sino una parte necesaria de la misma, que contribuye a hacerla avanzar.

[...] en esos dos vieneses [Broch y Musil] la reflexión ya no se siente como un elemento excepcional, una interrupción; es difícil llamarla «digresión» porque, en esas novelas que piensan, esta sin cesar presente, incluso cuando el novelista cuenta una acción o cuando descubre un rostro (Kundera, 2005a, p. 90)

Aunque en algunos pasajes el pensamiento novelesco pueda participar de rasgos propios del ensayo y proponer una reflexión general sobre un tema, el conocimiento que desarrolla la novela no se limita a esos fragmentos ensayísticos, sino que se nutre, como hemos visto, de la construcción de los personajes (su punto de vista, su visión del mundo, sus valores).

[...] la reflexión novelesca, tal como la introdujeron Broch y Musil en la estética de la novela moderna, no tiene nada que ver con la de un científico o un filósofo; diría incluso que es intencionadamente afilosófica, incluso antifilosófica, es decir, ferozmente independiente de todo sistema de ideas preconcebidas; no juzga; no proclama verdades; se interroga, se sorprende, sondea; adquiere las más diversas formas: metafórica, irónica, hipotética, aforística, cómica, provocadora, fantasiosa; y sobre todo: jamás abandona el círculo mágico de la vida de los personajes; se nutre y se justifica por la vida de los personajes. (Kundera, 2005a, p. 90)

Como podemos ver, el ensayo específicamente novelesco se nutre de la teoría de Broch y de las obras de Musil, pero Kundera la adapta a su propia concepción compositiva y arquitectónica de la novela imponiendo el ideal polifónico, es decir, la creación de distintas voces y líneas temáticas que discurren simultáneas. Aun así, como nos recuerdan Paola Gambarota y Liisa Steinby, lo que separa la visión de Broch de la de Kundera es sobre todo la actitud escéptica de este último: un escepticismo presente tanto en su concepción del personaje, como del hombre y sus facultades gnoseológicas limitadas.

Pero ¿en qué consiste esta sabiduría? ¿Qué es la novela? Hay un admirable proverbio judío que dice: El hombre piensa, Dios ríe. Inspirándome en esta sentencia, me gusta imaginar que François Rabelais oyó 


\begin{abstract}
un día la risa de Dios y que así fue como nació la primera gran novela europea. Me complace pensar que el arte de la novela ha llegado al mundo como eco de la risa de Dios.

¿Por qué ríe Dios al observar al hombre que piensa? Porque el hombre piensa y la verdad se le escapa.

Porque cuanto más piensan los hombres, más lejano está el pensamiento de uno del pensamiento de los otros.

Y, finalmente, porque el hombre nunca es lo que cree ser. (Kundera, 2007, p. 186)
\end{abstract}

Broch, en sus escritos sobre la novela buscaba en ella una forma de conocimiento que fuera capaz de crear una ilusión de totalidad, que recuperara aquella unidad que el sistema de valores heredado de la cultura platónico-cristiana había tenido en algún momento y que se disgregaba en sistemas parciales ante su mirada. Kundera escribe consciente de que esa unidad perdida es falaz y que el conocimiento que puede alcanzar la novela será siempre interrogativo, hipotético y, en última instancia, lúdico, pero no por ello menos válido para comprender el comportamiento humano y la existencia.

\title{
Referencias bibliográficas
}

BROCH, H. (1957). Briefe. Von 1929-1951. Zürich: Rhein-Verlag. (1974). Poesía e investigación. Barcelona: Barral. (1975). Schriften zur Literatur 2. Frankfurt am Main: Suhrkamp. (1986). Die Schlafwandler. Frankfurt am Main: Suhrkamp.

DURZAK, M. (1978). Hermann Broch. Dichtung und Erkenntnis. Studien zum dichterischen Werk. Stuttgart: Kohlhammer.

DURZAK, M y SCHLANT, E. (Eds.) (1972). Hermann Broch. Perspektiven der Forschung, München: Wilhelm Fink Verlag.

GABOLDE, I. (2010). La lecture comme pratique cognitive et devoir d'éveil. Frankfurt am Main: Peter Lang.

GAMBAROTA, P. (1999). Notizen zu Kunderas Broch-Lektüre. Germanoslavica : Zeitschrift für germano-slawische Studien, 6, (11), 47-59.

KUNDERA, M. (1986). L'art du roman. Paris: Gallimard.

_ (1993). Les testaments trahis. Paris: Gallimard. (2005). Le Rideau. Paris: Gallimard.

(2005a). El telon. Barcelona: Tusquets.

(2007). El arte de la novela. Barcelona, Tusquets.

(2007a). Los testamentos traicionados. Barcelona: Tusquets.

LÜTZELER, P. M. y KESSLER, M. (Eds.) (1987). Hermann Broch. Das dichterische Werk. Tübingen: Stauffenburg.

MANDELKOW, K. R. (1962). Herman Brochs Romantrilogie „Die Schlafwandler“. Gestaltung und Reflexion im modernen deutschen Roman. Heidelberg: Carl Winter Universitätsverlag.

MONDON, C. (2011). Écritures romanesques et philosophie. Pessac: Presses Universitaires de Bourdeaux.

NIETZSCHE, F. (2010). Fragmentos póstumos (1882-1885) Volumen III. Madrid: Tecnos. (2012). Más allá del bien y del mal. Madrid: Alianza editorial. 
RABATE, J.M. (1983). Le sourire du somnambule : de Broch à Kundera. Critique, 433-434, 505- 521. RITZER, M. (1988). Hermann Broch und die Kulturkrise im frühen 20. Jahrhundert. Stuttgart: J. B Metzler.

SAARILUOMA, L. (1999). Kundera, Broch und die Tradition des modernen Romans. Poetica, 30, (1/2), 179-199.

STEINBY, L. (née SAARILUOMA) (2013). Kundera and Modernity. West Lafayette, Indiana: Purdue University Press.

STEINECKE, H. (1968). Hermann Broch und der polyhistorische Roman. Studien zur Theorie und Technik eines Romantypus der Moderne. Bonn: H. Bouvier. 